\title{
Refractive Index and Strain Sensitivities of a Long Period Fiber Grating
}

\author{
Quandong HUANG ${ }^{1,2}$, Yongqin $\mathrm{YU}^{2,3^{*}}$, Zhilong OU ${ }^{1,2}$, Xue $\mathrm{CHEN}^{1,2}$, \\ Jishun WANG ${ }^{1,2}$, Peiguang YAN ${ }^{1,2}$, and Chenlin $\mathrm{DU}^{1,2}$ \\ ${ }^{1}$ College of Electronic Science and Technology, Shenzhen University, Shenzhen, 518060, China \\ ${ }^{2}$ Shenzhen Key Laboratory of Laser Engineering, Shenzhen, 518060, China \\ ${ }^{3}$ College of Physics Science and Technology, Shenzhen University, Shenzhen, 518060, China \\ ${ }^{*}$ Corresponding author: Yongqin YU E-mail: yuyq@szu.edu.cn
}

\begin{abstract}
A long period fiber grating (LPFG) fabricated upon the all-solid photonic bandgap fiber by $\mathrm{CO}_{2}$ laser irradiation was investigated, and its resonance wavelength was at $1335.76 \mathrm{~nm}$ with a modulation depth of $15 \mathrm{~dB}$ and a $3-\mathrm{dB}$ bandwidth of $2.6 \mathrm{~nm}$. We studied its strain, temperature, and index sensor characteristics, the strain sensitivity of $0.992 \mathrm{pm} / \mu \varepsilon$ was obtained by using linear fit, and the relationship between the refractive index and wavelength obeyed the distribution of quadratic function. Also, we demonstrated its temperature response was relatively insensitive $\left(21.51 \mathrm{pm} /{ }^{\circ} \mathrm{C}\right)$.
\end{abstract}

Keywords: Long period fiber grating, strain sensors, index sensors

Citation: Quandong HUANG, Yongqin YU, Zhilong OU, Xue CHEN, Jishun WANG, Peiguang YAN, et al., "Refractive Index and Strain Sensitivities of a Long Period Fiber Grating," Photonic Sensors, 2014, 4(1): 92-96.

\section{Introduction}

The optical fiber grating, such as fiber Bragg grating (FBG) $[1,2]$ and long period fiber grating (LPFG) [3], has been in great use in the field of optical communication and sensors. And the LPFG, consisting of a periodic change in the refractive index or the fiber geometry along the fiber length, possesses so many advantages, which has been widely used in sensing applications. Light is coupled from the core mode to the resonant co-propagation cladding modes that are absorbed by the coating causing the characteristic attenuation bands in the transmission spectrum [8]. Lots of methods have been used for fabricating the LPFG such as ultraviolet (UV) laser exposure [3], $\mathrm{CO}_{2}$ laser irradiation [4], and femtosecond laser exposure [5].
The LPFG has also been fabricated upon several kinds of fiber such as simple mode fiber, hollow-core photon bandgap fiber, and all-solid photon bandgap fiber $[6,7]$. In recent years, the LPFG has been attracting more and more academic's attention to research it for its wide applications in various physical parameter measurements such as temperature, bending, strain, refractive index, and twist sensitivity [8-18].

In this paper, we describe an LPFG all-solid photonic bandgap fiber (AS-PBF) fabricated by high-frequency $\mathrm{CO}_{2}$ laser pulse irradiation. The LPFG with a resonance wavelength of $1335.76 \mathrm{~nm}$ with a modulation depth of $15 \mathrm{~dB}$ and a $3-\mathrm{dB}$ bandwidth of $2.6 \mathrm{~nm}$ was obtained in experiments. Its stain, temperature, and refractive index sensitivities were studied. 


\section{Experimental setup}

The cross section captured by the optical microscope (Olympus BX51) of the AS-PBF is shown in Fig. 1. The AS-PBF with the cladding diameter of about $123 \mu \mathrm{m}$ and pitch ( $\Lambda$ ) between adjacent air holes of $9.3 \mu \mathrm{m}$ was used for fabricating the LPFG. Within the fiber, the diameters of the Ge-doped high-index rods surrounded by the F-doped low-index cladding were $d_{1}=4.06 \mu \mathrm{m}$ and $d_{2}=6.9 \mu \mathrm{m}$, respectively.

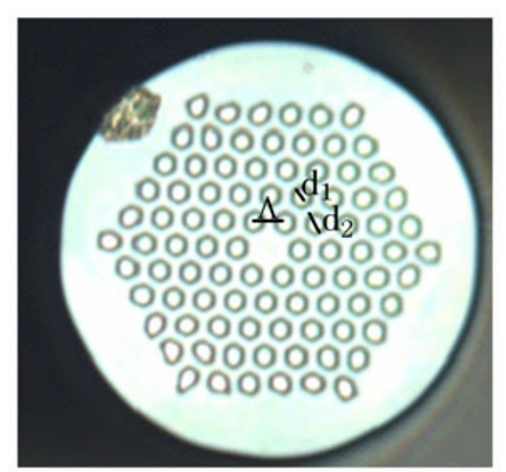

Fig. 1 Cross section of the AS-PBF.

From Fig. 2(a), the fabricated LPFG had a period

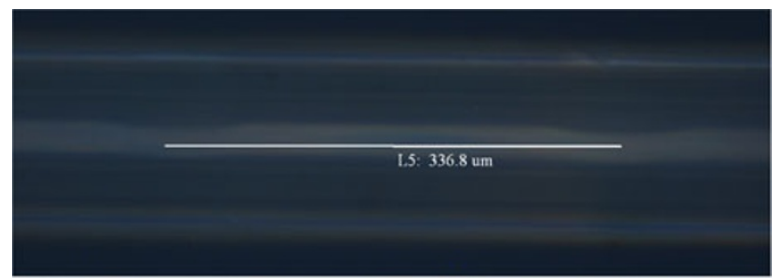

(a)

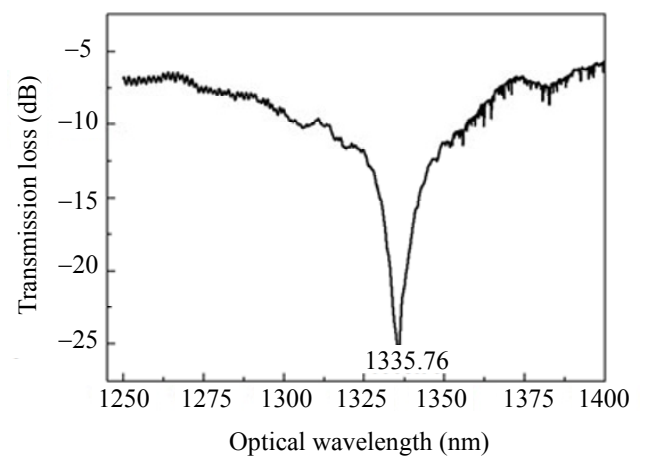

(b)

Fig. 2 Schematic of the fiber notch and transmission spectrum of the LPFG written on the AS-PBF: (a) fiber notch produced by $\mathrm{CO}_{2}$ laser and (b) transmission spectrum of the LPFG written on the AS-PBF. characteristic of about $330 \mu \mathrm{m}$, and its total length was $9.9 \mathrm{~mm}$. We added a polarization controller (PC) to adjust the depth of the transmission dip, and the transmission spectrum was also recorded by an optical spectrum analyzer (OSA, Yokogawa AQ6370B) with a super luminescent light-emitting diode (SLED) light source, which is shown in Fig. 2(b). The LPFG with a resonance wavelength of $1335.76 \mathrm{~nm}$, a modulation depth of $15 \mathrm{~dB}$, and a $3-\mathrm{dB}$ bandwidth of $2.6 \mathrm{~nm}$ was obtained in our experiments.

\section{Strain measurement}

The strain sensitivity of the LPFG was studied. The experimental setup for strain investigating is shown in Fig. 3. Two stages were used to hold and keep the LPFG straight: one stage was fixed, and the other was movable to provide the strain. The distance $L$ between two stages was set to $4 \mathrm{~cm}$.

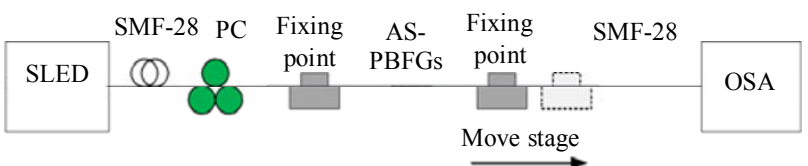

Fig. 3 Experimental setup for strain investigating.

The resonant matching condition of an LPFG can be described in (1), here $\lambda$ represents the resonant wavelength, $\Lambda$ represents the period of an LPFG, and $n_{\text {eff }}^{\text {core }} n_{\text {eff }}^{\text {clading }}$ are the effective mode indices of the core and cladding, respectively.

$$
\lambda=\left(n_{\text {eff }}^{\text {core }}-n_{\text {eff }}^{\text {clading }}\right) \Lambda .
$$

The relationship between the wavelength shift and stain [10] was demonstrated in (2) by a derivative with respect to axial strain $(\varepsilon)$ :

$$
\frac{d \lambda}{d \varepsilon}=\left(\frac{d n_{\mathrm{eff}}^{\text {core }}}{d \varepsilon}-\frac{d n_{\mathrm{eff}}^{\text {cladding }}}{d \varepsilon}\right) \Lambda+\left(n_{\mathrm{eff}}^{\text {core }}-n_{\mathrm{eff}}^{\text {cladding }}\right) \frac{d \Lambda}{d \varepsilon} .
$$

In Fig. 4, the sensitivity of strain was achieved to be $0.992 \mathrm{pm} / \mu \varepsilon$, which was about 1.223 times larger than that of the hollow-core photonic crystal fiber [11] and about 1.03 times larger than that of the modal interferometer [12], but about 0.354 times less sensitive than that in [10], negative correlation 
shows the interference dip moves toward the shorter wavelength (blue shift).

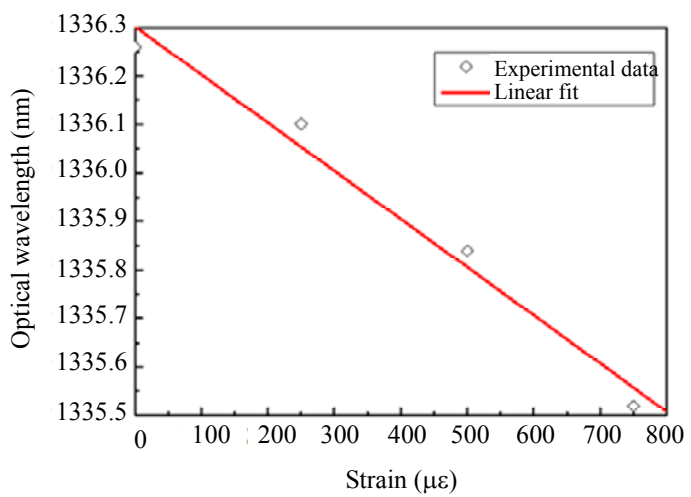

Fig. 4 Linear fit showing the relationship between the dip wavelength and strain.

\section{Temperature measurement}

The response of the LPFG to temperature was also studied by monitoring the shifts of the dip wavelength in a V-groove of a cylindrical temperature heater with a temperature range from $30{ }^{\circ} \mathrm{C}$ to $100{ }^{\circ} \mathrm{C}$, at $10{ }^{\circ} \mathrm{C}$ intervals. The previous research results in [13] demonstrated that the temperature response of the LPFG was weakly dependent on the polarization states of input light. Hence in our experiments, we adjusted the polarization controller in front of the LPFG to get the maximum modulation depth of $15 \mathrm{~dB}$, as shown in Fig. 2(a). Figure 5 shows the linear fit of the relationship between the dip wavelength and temperature $(\Delta \lambda / \Delta T)$, we got the relatively insensitive result of $-21.51 \mathrm{pm} /{ }^{\circ} \mathrm{C}$, but it is about

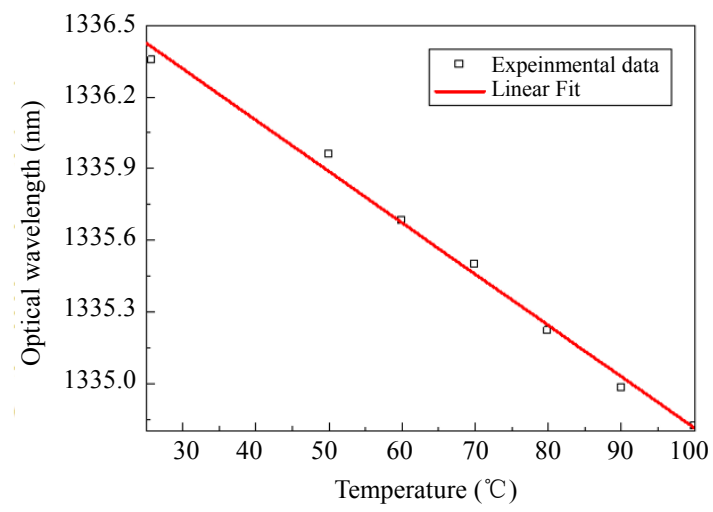

Fig. 5 Linear fit showing the relationship between the dip wavelength and temperature.
1.434 times larger than that in [11] and about 1.625 times larger than that of the photonic crystal fiber in the line Mach-Zehnder interferometer [12], because hollow-core photonic crystal fibers (HC-PCFs) used for the temperature sensor have a lower temperature sensitivity [16-18].

\section{Refractive index measurement}

We know from (3) that the refractive index difference between the core and cladding can be changed gradually when different surrounding refractive indices are added to the cladding of the LPFG, and $L$ in (3) represents the length of the LPFG. The effects of its sensitive characteristic can be investigated for sensing the surrounding medium's refractive index using the equation

$$
\Delta n_{\mathrm{eff}}=n_{\mathrm{eff}}^{\text {core }}-n_{\mathrm{eff}}^{\text {cladding }} \approx \frac{\lambda_{0}{ }^{2}}{\Lambda \cdot L} .
$$

The experimental setup for the surrounding refractive index investigation is shown in Fig. 6.

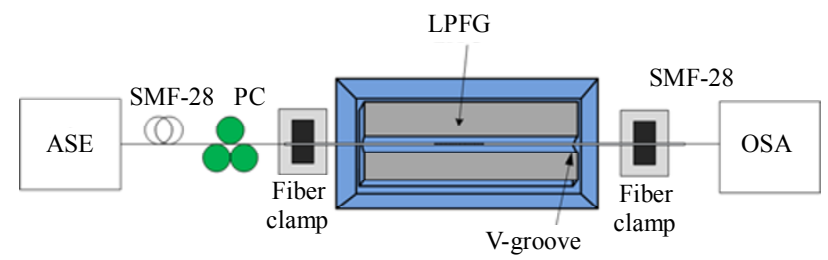

Fig. 6 Experimental setup for the surrounding refractive index investigation.

Different refractive index liquids were used in our experiment, and the dip wavelength of transmission spectrum shifts with the change in the refractive index, as shown in Fig. 7.

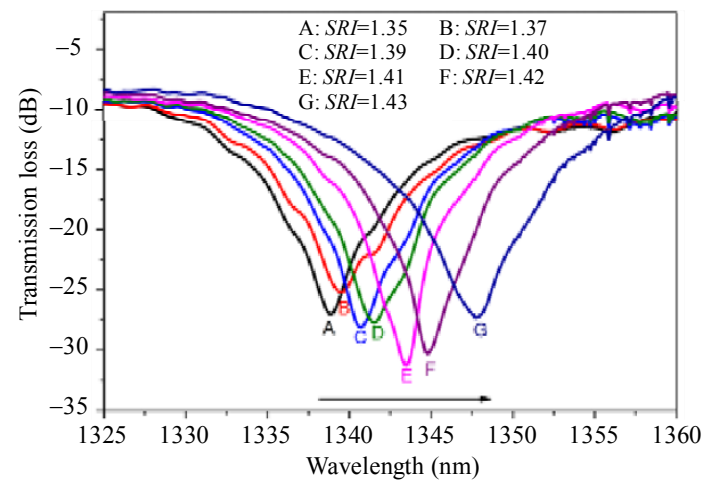

Fig. 7 Relationship between the dip wavelength and different refractive indices: each capital letter represents the solution with the different refractive index. 
At last, we used the polynomial fit to the experimental data by just three orders, as shown in Fig. 8, and the R-square factor of 0.99892 was achieved. Refractive index changes can be divided in three regions.

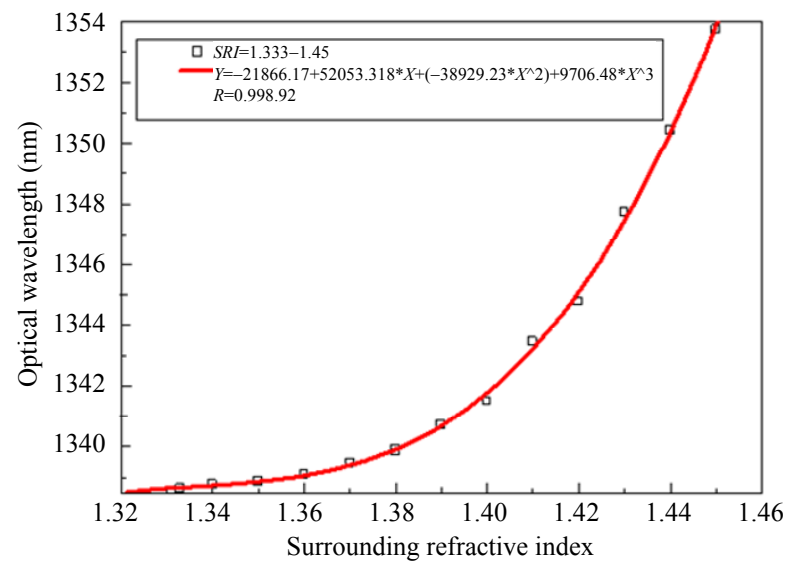

Fig. 8 Polynomial fit showing the relationship between the dip wavelength and refractive index.

In one of these regions, the surrounding refractive index (SRI) lies between 1.33 and 1.36, and the corresponding dip wavelength change is plotted in Fig.9.

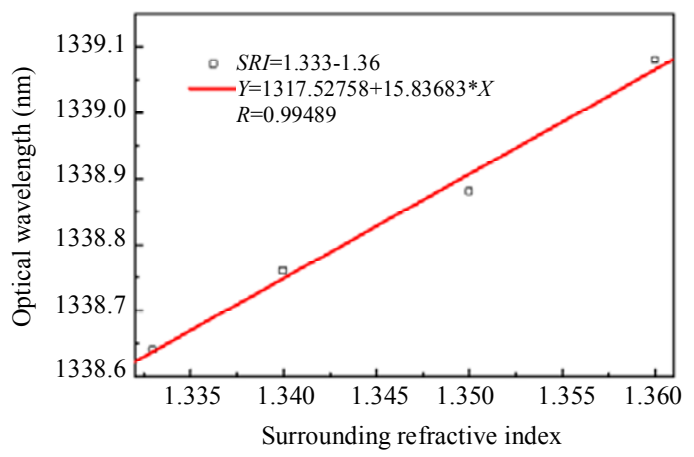

Fig. 9 Refractive index response in the 1.33-1.36 region.

The response slopes of $15.84 \mathrm{~nm} / \mathrm{RIU}$, $69.6 \mathrm{~nm} / \mathrm{RIU}$, and $262.4 \mathrm{~nm} / \mathrm{RIU}$ were obtained in $S R I=1.33-1.36,1.37-1.4$, and $1.41-1.45$ regions, respectively, as shown in Figs. 10 and 11.

Their R-square linear fits are 0.99489, 0.99105, and 0.99148 , respectively. The relationship is fitted by (4):

$$
\begin{aligned}
Y= & -21866.17+52053.31 * X-38929.23 * X^{\wedge} 2 \\
& +9706.48 * X^{\wedge} 3 .
\end{aligned}
$$

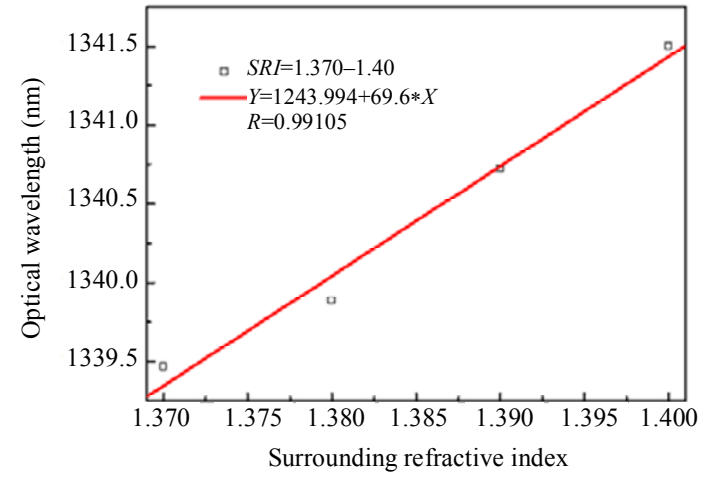

Fig. 10 Refractive index response in the 1.3-1.4 region.

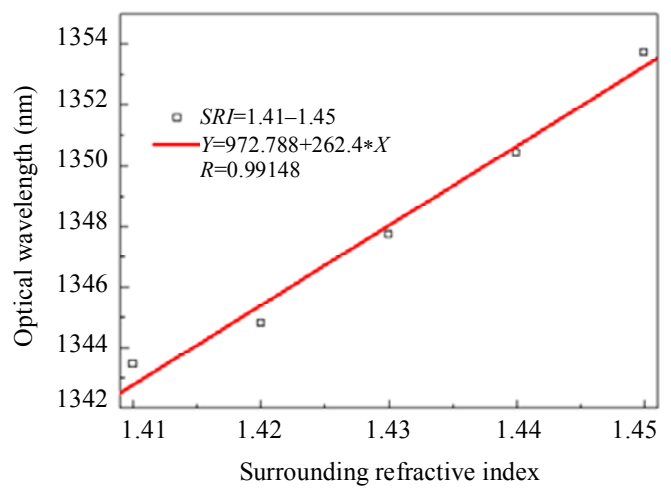

Fig. 11 Refractive index response in the $1.41-1.45$ region.

\section{Conclusions}

In conclusion, we fabricated an LPFG upon the all-solid photonic bandgap fiber by $\mathrm{CO}_{2}$ laser irradiation and obtained the strain sensitivity of $0.992 \mathrm{pm} / \mu \varepsilon$. The relationship between the refractive index and wavelength obeyed the distribution of the quadratic function. Also, we demonstrated its temperature response was relatively insensitive $\left(21.51 \mathrm{pm} /{ }^{\circ} \mathrm{C}\right)$.

\section{Acknowledgment}

This work was supported by the National Natural Science Foundation of China (NSFC) under Grants No. 61275125, 61007054, 61308055, National High Technology Research and Development Program of China under Grant No. 2013AA031501 \& 2012AA041203 Shenzhen Science and Technology Project (NO. JC201005280473A， JC201104210019A, ZDSY20120612094753264, JCYJ20130326113421781) 
and Specialized Research Fund for the Doctoral Program of Higher Education (SRFDP, 20124408120004).

Open Access This article is distributed under the terms of the Creative Commons Attribution License which permits any use, distribution, and reproduction in any medium, provided the original author(s) and source are credited.

\section{References}

[1] K. O. Hill, B. Malo, F. Bilodeau, D. C. Johnson, and J. Albert, "Bragg gratings fabricated in monomode photosensitive optical fiber by UV exposure through a phase mask," Applied Physics Letters, 1993, 62(10): 1035-1037.

[2] Y. J. Rao, "In-fiber Bragg grating sensors," Measurement Science \& Technology, 1997, 8(4): 355-375.

[3] A. M. Vengsarkar, P. J. Lemaire, J. B. Judkins, V. Bhatia, T. Erdogan, and J. E. Sipe, "Long-period fiber grating as band-rejection filters," Journal of Lightwave Technology, 1996, 14(1): 58-65.

[4] Y. P. Wang, D. N. Wang, W. Jin, Y. J. Rao, and G. D. Peng, "Asymmetric long period fiber gratings fabricated by use of $\mathrm{CO}_{2}$ laser to carve periodic grooves on the optical fiber," Applied Physics Letters, 2006, 89(15): 151105-1-151105-3.

[5] Y. Kondo, K. Nouchi, T. Mitsuyu, M. Watanabe, P. G. Kazansky, and K. Hirao, "Fabrication of long-period fiber gratings by focused irradiation of infrared femtosecond laser pulses," Optics Letters, 1999, 24(10): 646-648.

[6] Y. P. Wang, C. R. Liao, X. Y. Zhong, J. T. Zhou, Y. J. Liu, Z. R. Li, et al., "Long period fiber gratings writing in photonic crystal fber by use of $\mathrm{CO}_{2}$ laser," Photonic Sensors, 2013, 3(3): 193-201.

[7] J. Ju and W. Jin, "Long period gratings in crystal fibers," Photonic Sensor, 2012, 2(1): 65-70.

[8] T. Erdogan, "Cladding-mode resonances in short-and long-period fiber grating filters," Journal of the Optical Society of America A, 1997, 14(8): 1760-1773.

[9] Y. J. Rao, Y. P. Wang, Z. L. Ran, and T. Zhu, "Novel fiber-optic sensors based on long-period fiber gratings written by high-frequency $\mathrm{CO}_{2}$ laser pulses," Journal of Lightwave Technology, 2003, 21(5):1320-1327.

[10] J. Villatoro, V. Finazzi, V. P. Minkovich, V. Pruneri, and G. Badenes, "Temperature-insensitive photonic crystal fiber interferometer for absolute strain sensing," Applied Physics Letters, 2007, 91(091109): 091109-1-091109-3.

[11] G. Kim, T. Cho, K. Hwang, K. Lee, K. S. Lee, Y. G. Han, et al., "Strain and temperature sensitivities of an elliptical hollow-core photonic bandgap fiber based on Sagnac interferometer," Optics Express, 2009, 17(4): 2481-2486.

[12] S. H. Aref, R. Amezcua-correa, J. P. Carvalho, O. Frazão, P. Caldas, J. L. Santos, et al., "Modal interferometer based on hollow-core phonic crystal fiber for strain and temperature measurement," Optics Express, 2009, 17(21): 18669-18675.

[13] J. R. Zheng, Y. Q. Yu, Z. L. Ou, P. G. Yan, J. S. Wang, $\mathrm{X}$. Chen, et al., "Bending characteristics of long period fiber grating fabricated upon all-solid photonic bandgap fiber by $\mathrm{CO}_{2}$ laser," Optical Engineering, 2013, 52(6): 065002-1-065002-6.

[14] J. R. Zheng, P. G. Yan, Y. Q. Yu, Z. L. Ou, J. S. Wang, $\mathrm{X}$. Chen, et al., "Temperature and index insensitive strain sensor based on a photonic crystal fiber in line Mach-Zehnder interferometer," Optics Communications, 2013, 297(15): 7-11.

[15] H. Xuan, W. Jin, M. Zhang, J. Ju, and Y. Liao, "In-fiber polarimeters based on hollow-core photonic bandgap fibers," Optics Express, 2009, 17(15): 13246-13254.

[16] P. Zu, C. C. Chan, Y. Jin, T. Gong, Y. Zhang, L. H. Chen, et al., "A temperature-insensitive twist sensor by using low birefringence photonic crystal fiber based Sagnac interferometer," IEEE Photonics Technology Letters, 2011, 23(13): 920-922.

[17] Q. Shi, F. Lv, Z. Wang, L. Jin, J. J. Hu, Z. Liu, et al., "Environmentally stable Febry-Pérot-type strain sensor based on hollow core photonic bandgap fiber," IEEE Photonics Technology Letters, 2008, 20(4): 237-239.

[18] Q. Shi, Z. Huang, L. Jin, Y. Li, H. Zhang, F. Lu, et al., "A hollow-core photonic crystal fiber cavity based multiplexed Fabry-Pérot interferometric strain sensor system," IEEE Photonics Technology Letters, 2008, 20(15): 1329-1331. 